Desi gn of quasi-opt i cal systems converting a gyr ot ron out put i nt o a Gaussi an- I i ke beam

\begin{tabular}{|l|l|}
\hline 著者 & $\begin{array}{l}\text { OGAWA I, I DEHARA T, PEREYASLAVETS M L, } \\
\text { KASPAREK W }\end{array}$ \\
\hline $\begin{array}{l}\text { j our nal or } \\
\text { publ i cat i on t i t l e }\end{array}$ & I nt er nat i onal J our nal of El ect r oni cs \\
\hline vol une & 87 \\
\hline number & 7 \\
\hline page $\mathrm{r}$ ange & $865-877$ \\
\hline year & $2000-07$ \\
\hline URL & ht t p: //hdl . handl e. net /10098/1539 \\
\hline
\end{tabular}




\title{
Desigm of quasi-optical systems converting a gyrotron output into a Gaussian-like beam
}

\author{
1. OGAWA* $\uparrow$, T. IDEHARA $\ddagger$ M. L. PEREYASLAVETS $\dagger$ and \\ W. KASPAREK $\S$
}

The quasi-optical systems designed by Gaussian optics can convert the $\mathrm{TE}_{06}$ mode output $(f=388 \mathrm{GHz})$ of a submillimetre wave gyrotron into a Gaussian-like beam. The linearly polarized main beam, with an elliptical cross-section produced by a quasi-optical antenna, is converted into a well-collimated beam with a circular cross-section by a system consisting of two parabolic cylinder mirrors or of a parabolic cylinder mirror and a special mirror with focal lengths different in two dimensions.

\section{Introduction}

Electromagnetic waves in the submillimetre wavelength range have applications in many fields including plasma diagnostics, material processing, material physics, astronomy and biophysics. Some applications, such as plasma scattering measurements (see, for example, Terumichi et al. 1984, Fekete et al. 1994, Suvorov et ai. 1997), need more intense waves than are presently available.

High frequency gyrotrons are the most promising candidates for delivering intense waves ranging from several hundred watts up to several kilowatts (Zaytsev et al. 1974, Spira-Hakkarainen et al. 1990, Idehara et al. 1995). In addition, gyrotrons have the advantage of tuning the output frequency by selecting the operating mode.

To be used as a radiation power source, the gyrotron output should be converted into a Gaussian-like beam because a gyrotron produces a $\mathrm{TE}_{m n}$ waveguide mode whose radiation pattern does not correspond to usual requirements.

A quasi-optical antenna (Vlasov and Orlova 1974) converts a gyrotron output into a linearly polarized beam whose far-field consists of a main beam with an elliptical cross-section and additional sidelobes. Since the ellipticity of the crosssection depends on the gyrotron output mode, it is important to convert the elliptical cross-section into a circular one. This is a major obstacle in developing a conversion system suitable for a frequency-tunable gyrotron.

The elliptical cross-section can be converted into a circular one by employing a system of two parabolic cylinder mirrors, or a parabolic cylinder mirror and a special mirror with focal lengths different in two dimensions. The shapes and arrangement of the mirrors are determined by assuming a bi-Gaussian beam. Such an assumption

* Corresponding author. e-mail: d891084@icpc00.icpc.fukui-u.ac.jp

$\uparrow$ Faculty of Engineering, Fukui University, Fukui 910 8507, Japan.

$\$$ Research Center for Development of Far-Infrared Region, Fukui University, Fukui 910-8507, Japan.

§Institut für Plasmaforschung, Universität Stuttgart, Pfaffenwaldring 31, D-70569 Stuttgart, Germany. 
is valid as long as we consider the main beam produced by the quasi-optical antenna in the far-field (Ogawa et al. 1999b).

In order to compare the two systems, we will design two types of conversion system for the $\mathrm{TE}_{06}$ mode output $(f=388 \mathrm{GHz})$ of the Gyrotron $\mathrm{FU}$ II (Idehara et al. 1992). This paper compares the qualities of the beams produced by the two systems.

\section{Treatment of the beam produced by an himge source as a bi-Gaussian beam}

The first element in the quasi-optical system is the quasi-optical antenna (figure 1), consisting of a circular waveguide (internal radius $a_{w}=14 \mathrm{~mm}$ ) with a step-cut and a parabolic reflector (focal length $f_{p}=21.75 \mathrm{~mm}$ ). The antenna converts the gyrotron output ( $\mathrm{TE}_{06}$ mode, $f=388 \mathrm{GHz}$, Brillouin angle $\alpha=9.93^{\circ}$ ) into a linearly polarized beam. Its electric and magnetic fields are parallel to the $x$-and $y$-directions, respectively.

Radiation reflected from the parabolic reflector of the quasi-optical antenna is treated as if it comes from a plane image source lying behind the parabolic reflector (Wada and Nakajima 1986, Brand et al. 1990).

In the design of the systems (figures 2 and 3), the image source is located in such a way that the beam with polarization in the $x$-direction propagates along the $z$-axis. The intensity profiles of the beam are calculated using the Huygens equation (Ogawa et al. 1997). The intensity profiles at the first mirror (mirror ml) are shown in figure 4. The beam produced by the image source consists of a main beam and additional sidelobes. In order to improve the beam quality, the size of the mirror $\mathrm{ml}$ is selected so as to truncate the sidelobes.

The distance between the image source and the mirror $\mathrm{ml}$ is large enough $(7000 \mathrm{~mm})$ to ensure that the spot sizes of the main beam are accurately given by assuming a bi-Gaussian beam whose waist $\left(w_{0 x}=38.7 \mathrm{~mm}\right.$ in the $x$-direction, $w_{0 y}=25.2 \mathrm{~mm}$ in the $y$-direction) is located at the centre of the image source (Ogawa et al. 1999a). The intensity of the bi-Gaussian beam is given by

$$
I=\frac{2 P_{0}}{\pi w_{x} w_{y}} \exp \left(-\frac{2 x^{2}}{w_{x}^{2}}\right) \exp \left(-\frac{2 y^{2}}{w_{y}^{2}}\right)
$$

where $w_{x}$ and $w_{y}$ are the spot sizes of the bi-Gaussian beam in the $x$ - and $y$-directions, respectively, and $P_{0}$ is the total beam power. The spot sizes correspond to the half-widths of the $-8.69 \mathrm{~dB}$ (or $\mathrm{e}^{-2}$ ) contours.
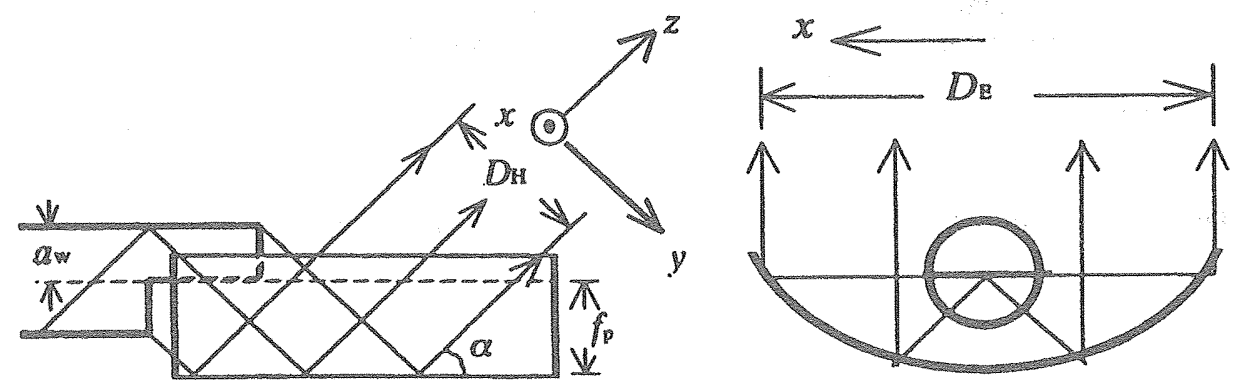

Figure 1. Quasi-optical antenna. The $x$-and $y$-directions correspond to the directions of electric and magnetic fields, respectively. 

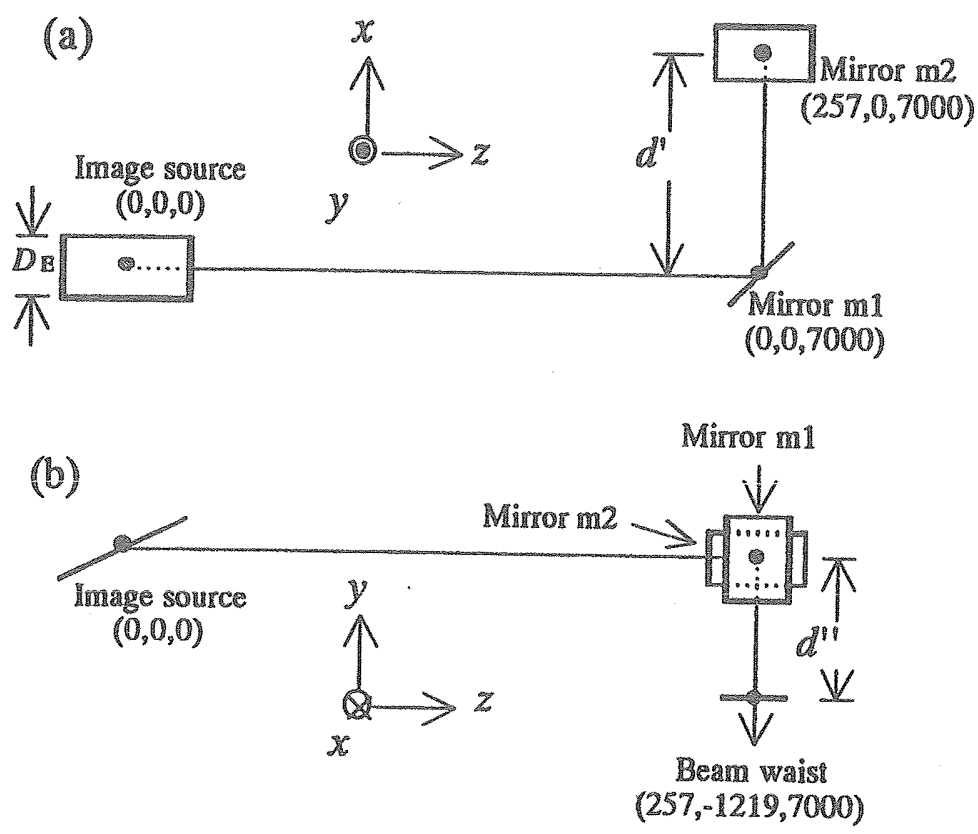

Figure 2. Quasi-optical system. (a) A plane image source is used for the calculation of the subsequent radiation patterns of the quasi-optical antema. The mirror $\mathrm{ml}$ is a parabolic cylinder whose focal axis is located in the $x-z$ plane. The mirroa focuses the beam in the $y$-direction. (b) The mirror $\mathrm{m} 2$ is a parabolic cylinder whose focal axis is located in the $x-y$ plane. The mirror focuses the beam in the $z$-direction.

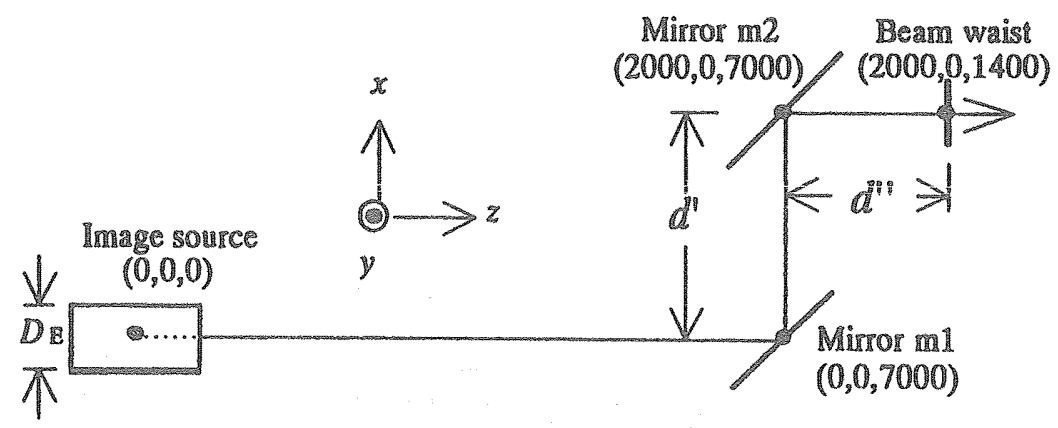

Figure 3. Quasi-optical system. A plane image source is used for the calculation of the subsequent radiation patterns of the quasi-optical antenna. The mirror $\mathrm{ml}$ is a parabolic cylinder whose focal axis is located in the $x-z$ plane. The mirror focuses the beam in the $y$-direction. The mirror $\mathrm{m} 2$ is a special one with focal lengths different in two dimensions.

The complex beam parameters $q_{x}$ and $q_{y}$ in the $x$ - and $y$-directions, defined by

$$
\left.\begin{array}{c}
\frac{1}{q_{x}}=\frac{1}{R_{x}}-\mathrm{j} \frac{\lambda}{\pi w_{x}^{2}} \\
\frac{1}{q_{y}}=\frac{1}{R_{y}}-\mathrm{j} \frac{\lambda}{\pi w_{y}^{2}}
\end{array}\right\}
$$




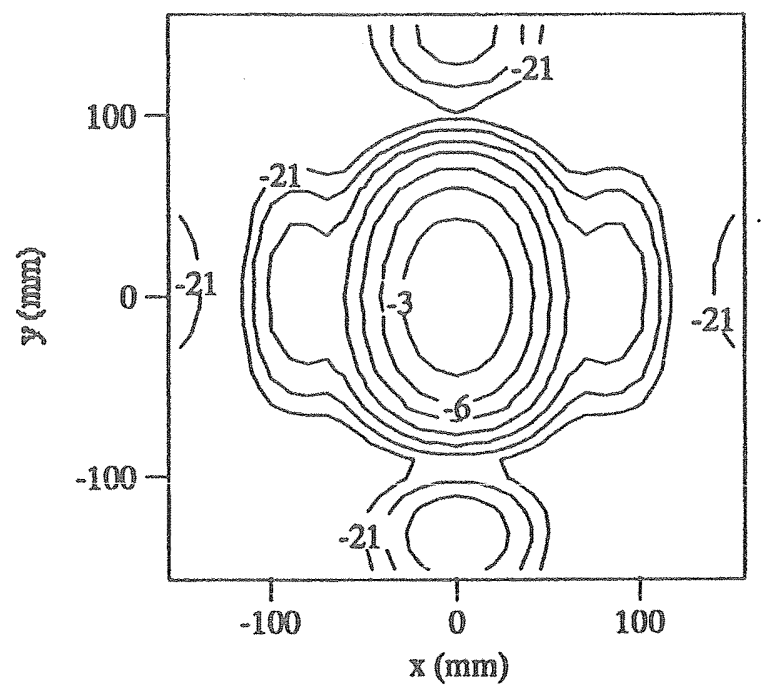

Figure 4. Calculated intensity contours at mirror $\mathrm{ml}$. Contours are in decibels relative to the intensity maximum.

are convenient parameters to treat the beam propagation and the focusing due to the focusing element, where $\mathbb{R}_{x}$ and $R_{y}$ are the curvature radii of the wave fronts in the $x$ and $y$-directions, respectively.

At the beam waist, $R_{x}, R_{y} \rightarrow \infty$. Therefore, the complex beam parameters are

$$
\left.\begin{array}{l}
q_{0 x}=\mathrm{j} \frac{\pi w_{0 x}^{2}}{\lambda} \\
q_{0 y}=\mathrm{j} \frac{\pi w_{0 y}^{2}}{\lambda}
\end{array}\right\}
$$

The complex beam parameters change when the beam propagates or is focused by any element. The complex beam parameters $q_{x}$ and $q_{y}$ change to

$$
\left.\begin{array}{l}
q_{x}^{\prime}=q_{x}+d \\
q_{y}^{\prime}=q_{y}+d
\end{array}\right\}
$$

after propagating a distance $d$. After the focusing element, they change to new values given by

$$
\left.\begin{array}{l}
\frac{1}{q_{x}^{\prime}}=\frac{1}{q_{x}}-\frac{1}{f_{x}} \\
\frac{1}{q_{y}^{\prime}}=\frac{1}{q_{y}}-\frac{1}{f_{y}}
\end{array}\right\}
$$

where $f_{x}$ and $f_{y}$ are the focal lengths of a focusing element in both directions.

\section{Design of the quasi-optical systems}

We have designed two types of system (System I and System II) to produce a collimated bean with a waist size of $5 \mathrm{~mm}$. System I (figure 2) consists of a quasi- 
optical antenna and two parabolic cylinder mirrors. It is easy to manufacture the mirrors, because they are simple in form. In addition to the quasi-optical antenna and the parabolic cylinder mirror, System II (figure 3) uses a special mirror with focal lengths different in two dimensions.

In order to remove the sidelobes, the size of mirror $\mathrm{mll}$ is determined as $144 \mathrm{~mm}$ in the $x$-direction and $195 \mathrm{~mm}$ in the $y$-direction. On the other hand, mirror $\mathrm{m} 2$ is wide enough to avoid any diffraction loss due to beam truncation.

The design is accomplished by converting the complex beam parameters $q_{0 x}$ and $q_{0 y}\left(q_{0 x} \neq q_{0 y}\right)$ at the image source into the two-dimensionally equal complex beam parameter $q_{0^{\prime}}$ at the beam waist according to equations (4) and (5). The values of $q_{0 x}$ and $q_{0 y}$ are obtained using equation (3) with $w_{0 x}=38.7 \mathrm{~mm}$ and $w_{0 y}=25.2 \mathrm{~mm}$. The value of $q_{0^{\prime}}$ is also obtained using equation (3) with $w_{0^{\prime}}=5 \mathrm{~mm}$.

As can be seen from equation (2), we need two procedures to equalize the twodimensionally different complex parameters $\left(q_{0 x} \neq q_{0 y}\right)$ into the same value $q_{0^{\prime}}$, namely, the coincidence of the spot sizes and of the curvature radii of the wave fronts. As can be seen from equations (4) and (5), the former is achieved by focusing and propagation, whereas the latter is achieved by focusing only.

\subsection{System I}

The mirror $m 1$ focuses the beam in the $y$-direction and the mirror $m 2$ focuses the beam in the $z$-direction (figure 2). The focal length $f_{1 y}$ of mirror $\mathrm{ml}$ and the propagation path $d\left(=d^{\prime}+d^{\prime \prime}\right)$ from mirror $\mathrm{ml}$ to the beam waist can be determined by the waist size of the beam produced $\left(w_{0^{\prime}}=5 \mathrm{~mm}\right)$. As can be seen from equations (4) and (5), they are given by

$$
\left.\begin{array}{l}
\frac{1}{q_{1 y}^{\prime}}=\frac{1}{q_{1 y}}-\frac{1}{f_{1 y}} \\
q_{0^{\prime}}=q_{1 y}^{\prime}+d
\end{array}\right\}
$$

where $q_{1 y}$ and $q_{1 y}^{\prime}$ are the complex beam parameters at mirror $\mathrm{ml}$ and just after reflection by this mirror, respectively. If we notice that $q_{0^{\prime}}$ is a pure imaginary, $d$ is given by

$$
d=-\operatorname{Im}\left(q_{1 y}^{\prime}\right)
$$

As can be seen from equation (2), the coincidence of the spot sizes at mirror $m 2$ is expressed by

$$
\operatorname{Im}\left(\frac{1}{q_{2 z}}\right)=\operatorname{Im}\left(\frac{1}{q_{2 y}}\right)
$$

where $q_{2 z}$ and $q_{2 y}$ are the complex beam parameters in the $z$ - and $y$-directions at mirror $\mathrm{m} 2$. Because the beam propagates in the opposite $y$-direction after its reflection from mirror $\mathrm{ml}$, the subscript $2 x$ of the complex beam parameter $q$ is replaced by $2 z$. The propagation path $d^{\prime}$ from mirror $\mathrm{ml}$ to mirror $\mathrm{m} 2$ is obtained by equation (8). Then, the propagation path $d^{\prime \prime}$ from mirror $\mathrm{m} 2$ to the beam waist is given by

$$
d^{\prime \prime}=d-d^{\prime}
$$


The focal length $f_{2 z}$ of mirror $\mathrm{m} 2$ can be determined by the condition of the coincidence of the curvature radii of the wave fronts. This is expressed by

$$
\frac{1}{q_{2 z}}-\frac{1}{f_{2 z}}=\frac{1}{q_{2 y}}
$$

The results thus obtained are listed in table 1.

Mirror $m 1$ is a parabolic cylinder whose focal axis is located in the $x-z$ plane (figure 2). The value of $f_{1 y}$ is given by (Ogawa et al. 1999a)

$$
f_{1 y}=\frac{f_{y}}{\cos \theta_{y}}
$$

where $f_{y}$ is the focal length of the parabolic cylinder and $\theta_{y}$ is the incident angle of the beam. In the present case, because $\theta_{y}$ is $45^{\circ}, f_{y}$ is determined as $884.2 \mathrm{~mm}$.

Mirror $\mathrm{m} 2$ is also a parabolic cylinder whose focal axis is located in the $x-y$ plane. The focal length $f_{z}$ of the parabolic cylinder is determined in the same way $\left(f_{z}=789.8 \mathrm{~mm}\right)$.

As can be seen from equation (11), a parabolic cylinder offers the tunability of focal lengths by the variation of the angle of incidence. This feature offers the possibility to tune the size of the beam produced.

\subsection{System III}

Mirror $m 1$ focuses the beam in the $y$-direction and helps to coincide the twodimensional spot sizes at mirror $m 2$ (figure 3 ). This is also expressed by equation (8). Because, unlike in System 1 , the mirror $m 2$ focuses the beam two-dimensionally, the focal length $f_{1 y}$ of the mirror $\mathrm{ml}$ can be defined arbitrarily. If we select the propagation path $d^{\prime}=2000 \mathrm{~mm}$ from $\mathrm{ml}$ to $\mathrm{m} 2, f_{1 y}$ is given by

$$
\left.\begin{array}{l}
\frac{1}{q_{1 y}^{\prime}}=\frac{1}{q_{1 y}}-\frac{1}{f_{1 y}} \\
q_{2 y}=q_{1 y}^{\prime}+d^{\prime} \\
q_{2 z}=q_{1 x}+d^{\prime}
\end{array}\right\}
$$

where $q_{1 x}$ is the complex beam parameter at $\mathrm{ml}$ and $q_{1 y}$ and $q_{1 y^{\prime}}$ are those before and just after reflection by $\mathrm{m} 1$. Following the change of the beam propagation direction, the subscript $2 x$ of the complex beam parameter $q$ is replaced by $2 z$.

\begin{tabular}{|c|c|c|c|c|c|c|c|c|}
\hline \multicolumn{3}{|c|}{ Mirror ml } & \multicolumn{4}{|c|}{ Mirror $\mathrm{m} 2$} & \multicolumn{2}{|c|}{ Beam waist } \\
\hline$f_{1 y}$ & $w_{1 x}$ & $w_{1 y}$ & $d^{\prime}$ & $f_{2 z}$ & $w_{2 z}$ & $w_{2 y}$ & $d^{\prime \prime}$ & $w_{0}$ \\
\hline 1250 & 59.0 & 72.9 & 257.3 & 1117 & 60.2 & 60.2 & 1219 & 5.0 \\
\hline
\end{tabular}

The focal lengths $f_{2 y}$ and $f_{2 z}$ of mirror $\mathrm{m} 2$ can be determined by the waist size of the beam produced $\left(w_{0^{\prime}}=5 \mathrm{~mm}\right)$. They are given by

Table 1. The results obtained by Gaussian optics for System $1 . w_{1 x}$ and $w_{1 y}$ are the spot sizes at mirror $\mathrm{ml}, w_{2 z}$ and $w_{2 y}$ are the spot sizes at mirror $\mathrm{m} 2$. All dimensions in millimetres. 


$$
\left.\begin{array}{l}
\frac{1}{q_{2 x}^{\prime}}=\frac{1}{q_{2 z}}-\frac{1}{f_{2 z}} \\
\frac{1}{q_{2 y}^{\prime}}=\frac{1}{q_{2 y}}-\frac{1}{f_{2 y}} \\
q_{0^{\prime}}=q_{2 x}^{\prime}+d^{\prime} \\
q_{0^{\prime}}=q_{2 y}^{\prime}+d^{\prime \prime}
\end{array}\right\}
$$

where $d^{\prime \prime}$ is the propagation path from $\mathrm{m} 2$ to the beam waist. The results thus obtained are listed in table 2.

Mirror $m 1$ is a parabolic cylinder whose focal axis is located in the $x-z$ plane (figure 3). The value of $f_{1 y}$ is given by equation (11). In this case, since $\theta_{y}$ is $45^{\circ}$, the focal length of the parabolic cylinder is determined as $f_{y}=4594.2 \mathrm{~mm}$.

Mirror $\mathrm{m} 2$ has focal lengths different in two dimensions $\left(f_{2 z} \neq f_{2 y}\right)$. The point

\begin{tabular}{|c|c|c|c|c|c|c|c|c|c|}
\hline \multicolumn{3}{|c|}{ Mirror ml } & \multicolumn{5}{|c|}{ Mirror $\mathrm{m} 2$} & \multicolumn{2}{|c|}{ Beam waist } \\
\hline$f_{1 y}$ & $w_{1 x}$ & $w_{1 y}$ & $d^{\prime}$ & $f_{2 z}$ & $f_{2 y}$ & $w_{2 z}$ & $w_{2 y}$ & $d^{\prime \prime}$ & $w_{0^{\prime}}$ \\
\hline 6497 & 59.0 & 72.9 & 2000 & 1271 & 1458 & 69.1 & 69.1 & 1400 & 5.0 \\
\hline
\end{tabular}
$\mathbb{P}(x, y, z)$ on $\mathrm{m} 2$ (figure 5) is given by (Ogawa et al. 1999b)

$$
\left.\begin{array}{l}
\left(d_{1}-d_{1 c}\right)+\left(d_{2}-d_{2 c}\right)=0 \\
d_{1}=\sqrt{\left(x-x_{1}\right)^{2}+\left(z-z_{1}\right)^{2}} \\
d_{2}=\sqrt{\left(x-x_{2}\right)^{2}+y^{2}+\left(z-z_{2}\right)^{2}} \\
d_{1 c}=\sqrt{\left(x_{c}-x_{1}\right)^{2}+\left(z_{c}-z_{1}\right)^{2}} \\
d_{2 c}=\sqrt{\left(x_{c}-x_{2}\right)^{2}+\left(z_{c}-z_{2}\right)^{2}}
\end{array}\right\}
$$

Table 2. The results obtained by Gaussian optics for System II. $w_{1 x}$ and $w_{1 y}$ are the spot sizes at mirror $\mathrm{ml}, w_{2 z}$ and $w_{2 y}$ are the spot sizes at mirror $\mathrm{m} 2$. All dimensions in millimetres.

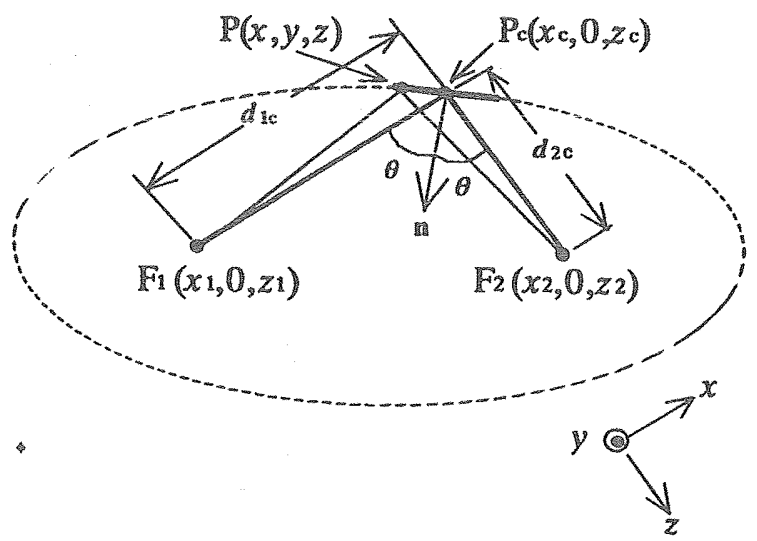

Figure 5. Geometry of the mirror $\mathrm{m} 2$. 
The value of $d_{1}$ is the distance between the point $\mathbb{F}_{1}\left(x_{1}, 0, z_{1}\right)$ and the point $\mathbb{P}$, and that of $d_{2}$ is the distance between $\mathbb{F}_{2}\left(x_{2}, 0, z_{2}\right)$ and $\mathbf{P}$. The values of $d_{1 c}$ and $d_{2 c}$ are those between $\mathbb{F}_{1}, \mathbb{F}_{2}$ and the mirror centre $\mathbb{P}_{c}\left(x_{c}, 0, z_{c}\right)$, respectively. The focal lengths $f_{2 y}$ and $f_{2 z}$ are given by

$$
\left.\begin{array}{l}
\frac{1}{f_{2 z}}=\frac{1}{d_{1 c}}+\frac{1}{d_{2 c}} \\
\frac{1}{f_{2 y}}=\frac{1}{d_{2 c}}
\end{array}\right\}
$$

If we select $d_{1 c}=9900 \mathrm{~mm}, d_{2 c}=1458 \mathrm{~mm}$, the mirror satisfies the conditions $\left(f_{2 z}=1271 \mathrm{~mm}, f_{2 y}=1458 \mathrm{~mm}\right)$ obtained by Gaussian optics. The focal points and the mirror centre fitting the system shown in figure 3 are $\mathbb{F}_{1}(-7900,0,7000)$, $\mathbb{F}_{2}(2000,0,8458)$ and $\mathbb{P}_{c}(2000,0,7000)$.

\section{Verification by the mumerical solution of the Huygens equation}

In order to verify the results obtained by Gaussian optics, we have carried out a numerical solution of the Huygens equation. First, the incident electromagnetic fields are calculated on the surface of the mirror $\mathrm{ml}$. The electromagnetic fields reflected by the mirror are obtained using the boundary conditions for a perfect conductor. The electromagnetic fields on the subsequent mirror $\mathrm{m} 2$ are obtained by repeatedly using the Huygens equation and the boundary condition employing the previously calculated results as the source.

\subsection{System I}

Calculated intensity contours at $\mathrm{m} 2$ are shown in figure 6 . The main beam, with an elliptical cross-section at $\mathrm{m} 1$ (figure 4 ), approaches an almost circular cross-section due to reflection from $\mathrm{ml}$ and is well collimated at the beam waist (figure 7), as is predicted by Gaussian optics. However, the beam has a triangular cross-section.

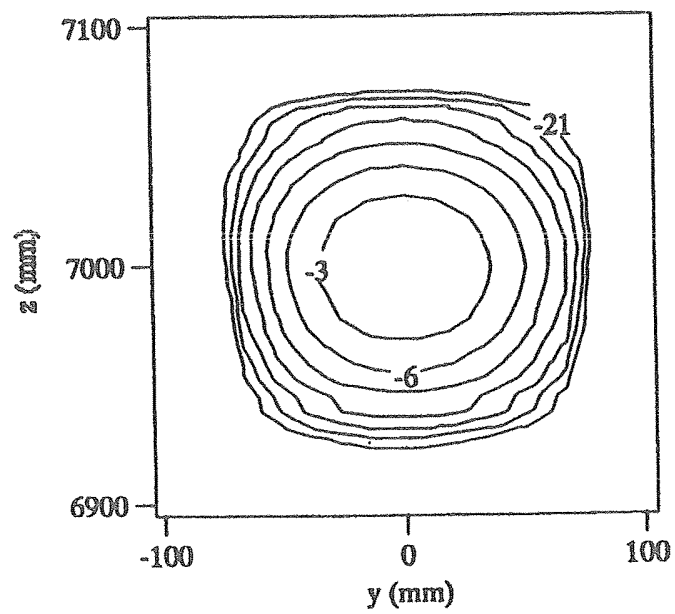

Figure 6. Calculated intensity contours at mirror $\mathrm{m} 2$. Contours are in decibels relative to the intensity maximum. 


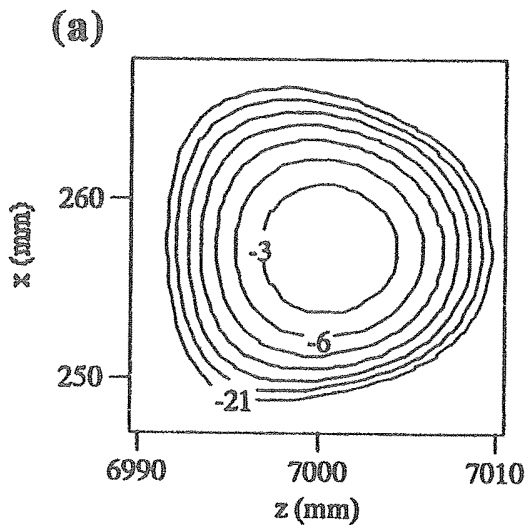

(b)

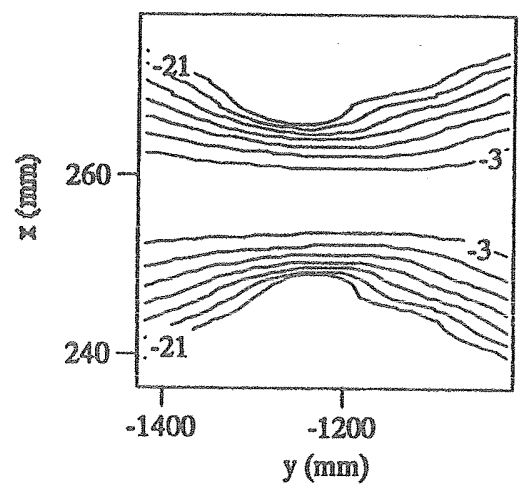

(c)

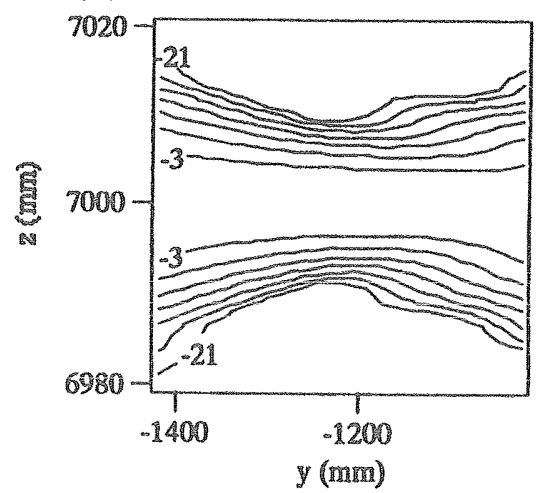

Figure 7. Calculated intensity contours at $(a)$ the beam waist, predicted by Gaussian optics contours are in decibels relative to the intensity maximum; $(b),(c)$ in the vicinity of the waist-contours are relative to the intensity along the $y$-axis.

The beam produced by the image source contains sidelobes in addition to the main beam, as can be seen in figure 4 . The quality of the beam is improved by truncating the sidelobes, which is done by limiting the size of $\mathrm{ml}$ to the optimum size (144 mm in the $x$-direction and $195 \mathrm{~mm}$ in the $y$-direction). As can be seen from the results (figure 8) for a wider size (179 $\mathrm{mm}$ in the $x$-direction and $221 \mathrm{~mm}$ in the $y$ direction), this attempt is effective. In spite of the truncation of the sidelobes, most of the power from the image source $(81.6 \%)$ is still reflected by $\mathrm{ml}$.

The results obtained from the calculations are listed in table 3 . The results obtained by the Gaussian optics approach (table 1) are in good agreement with the calculations.

\subsection{System II}

The main beam, with an elliptical cross-section at $\mathrm{ml}$ (figure 4), approaches an almost circular cross-section (figure 9) due to reflection from $\mathrm{ml}$. Calculated intensity contours in the vicinity of the beam waist are shown in figure 10 . This system can produce a well-collimated beam with a circular cross-section. As regards beam quality, this system is superior to System 1 . 
(अ)

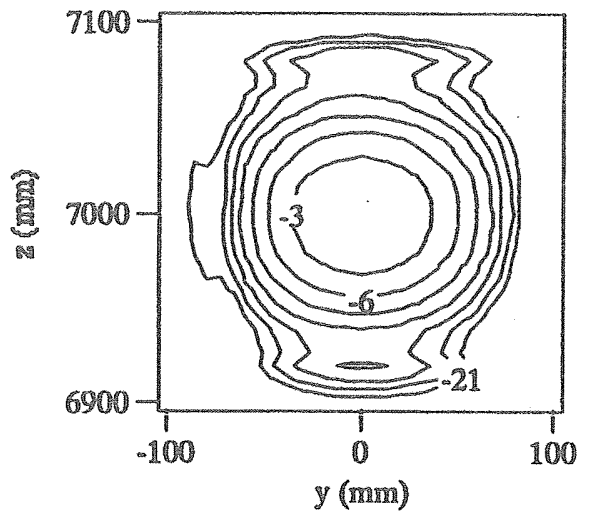

(b)

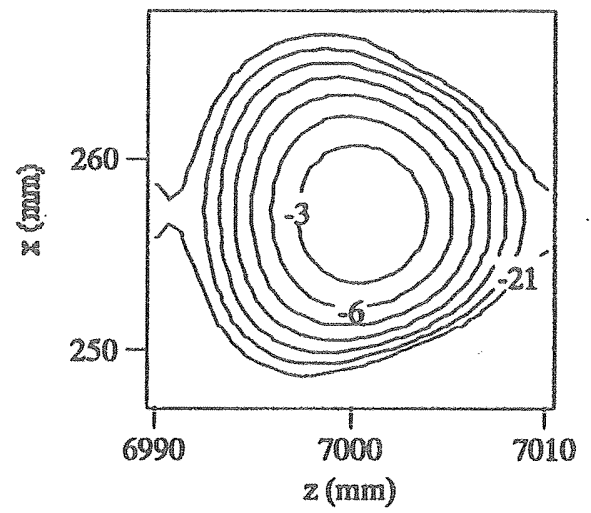

Figure 8. Calculated intensity contours at $(a)$ mirror $\mathrm{m} 2$ and $(b)$ the beam waist, predicted by Gaussian optics. Contours are in decibels relative to the intensity maximum.

\begin{tabular}{|c|c|c|c|c|c|c|c|}
\hline \multicolumn{2}{|c|}{ Mirror ml } & \multicolumn{2}{|c|}{ Mirror m2 } & \multicolumn{4}{|c|}{ Beam waist } \\
\hline$w_{1 x}$ & $w_{i y}$ & $w_{2 z}$ & $w_{2 y}$ & $d_{x}^{\prime \prime}$ & $d_{z}^{\prime \prime}$ & $w_{0 x^{\prime}}$ & $w_{z 0^{\prime}}$ \\
\hline 48.8 & 68.8 & 50.4 & 56.9 & 1200 & 1181 & 5.95 & 6.24 \\
\hline
\end{tabular}

Table 3. The resuits obtained by calculations for System 1. $w_{x 0^{\prime}}$ and $w_{z 0^{\prime}}$ are the spot sizes at the beam waist predicted by Gaussian optics. $d_{x}^{\prime \prime}$ and $d_{z}^{\prime \prime}$ are the positions where the beam has the waists. All dimensions in millimetres.

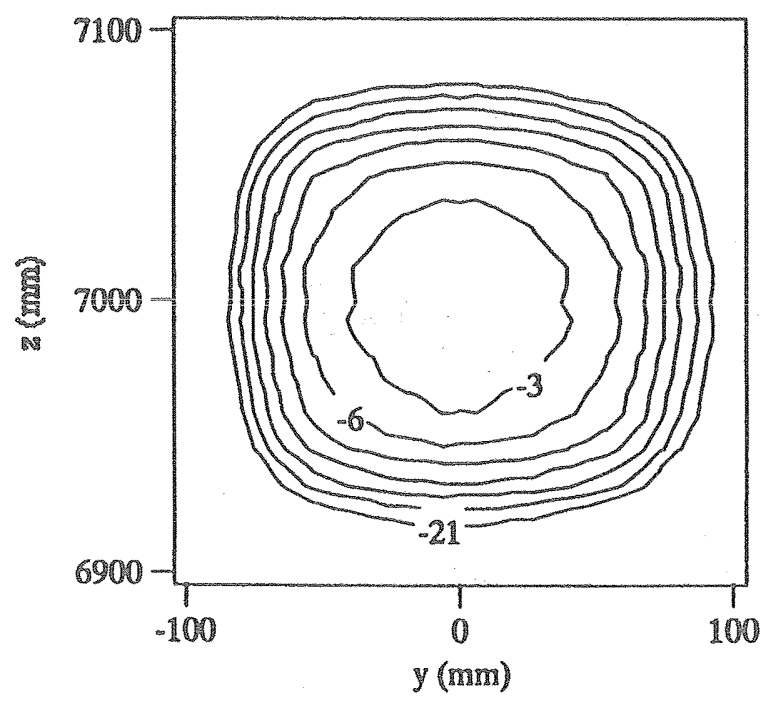

Figure 9. Calculated intensity contours at mirror $\mathrm{m} 2$. Contours are in decibels relative to the intensity maximum. 


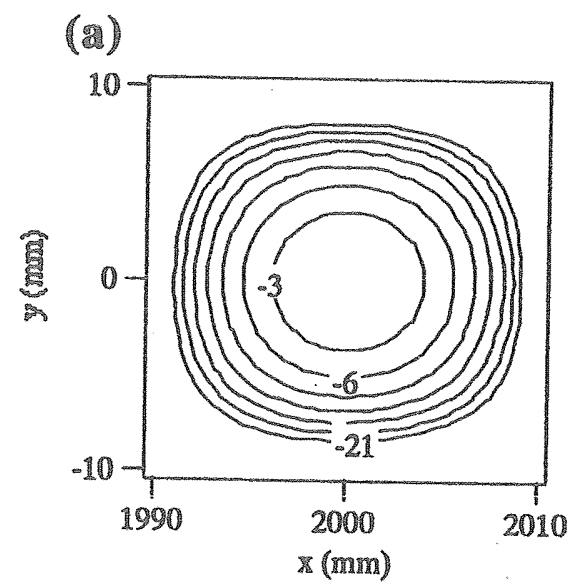

(ib)

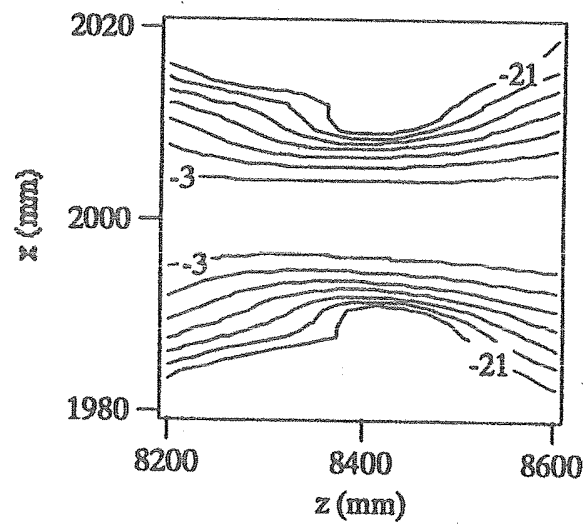

(c)

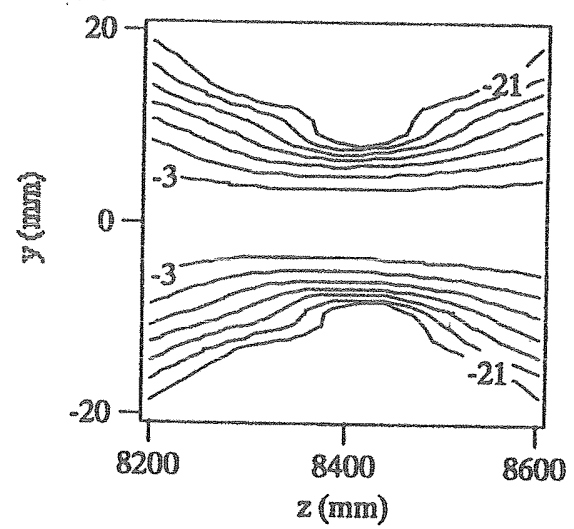

Figure 10. Calculated intensity contours at $(a)$ the beam waist, predicted by Gaussian optics-contours are in decibels relative to the intensity maximum: $(b),(c)$ in the vicinity of the waist-contours are relative to the intensity along the $z$-axis.

\begin{tabular}{|c|c|c|c|c|c|c|c|}
\hline \multicolumn{2}{|c|}{ Mirror ml } & \multicolumn{2}{|c|}{ Mirror m2 } & \multicolumn{4}{|c|}{ Beam waist } \\
\hline$w_{1 x}$ & $w_{1 y}$ & $w_{2 z}$ & $w_{2 y}$ & $d_{x}^{\prime \prime}$ & $d_{y}^{\prime \prime}$ & $w_{x 0^{\prime}}$ & $w_{y 0^{\prime}}$ \\
\hline 48.8 & 68.8 & 58.4 & 64.9 & 1372 & 1396 & 6.4 & 5.9 \\
\hline
\end{tabular}

Table 4 . The results obtained by calculations for System II. $w_{x 0^{\prime}}$ and $w_{y 0^{\prime}}$ are the spol sizes at the beam waist predicted by Gaussian optics. $d_{x}^{\prime \prime}$ and $d_{y}^{\prime \prime}$ are the positions where the beam has the waists. All dimensions in millimetres.

The spot sizes, the waist sizes and their positions obtained from the calculations are listed in table 4 for comparison. The results obtained by the Gaussian optics approach are in good agreement with these calculations.

\section{Conclusion}

The quasi-optical systems designed by Gaussian optics can convert the $\mathrm{TE}_{06}$ mode output $(f=388 \mathrm{GHz})$ of a submillimetre wave gyrotron into a Gaussian- 
like beam. The linearly polarized main beam, with an elliptical cross-section produced by a quasi-optical antenna, is converted into a well-collimated beam with a circular cross-section by a system consisting of two parabolic cylinder mirrors or a system consisting of a parabolic cylinder mirror and a special mirror with focal lengths different in two dimensions. The former is characterized by the possibility of tuning the size of the beam produced, in addition to its simplicity. The latter allows one to produce a high quality beam.

\section{Acknowledgments}

The authors would like to thank Dr D. Wagner, Universitatut Stuttgart, for valuable discussions and help.

This work was done as a collaboration between Fukui University, the National Institute for Fusion Science and the Universität Stuttgart, Germany. It has been supported by the Japan Society for the Promotion of Science (International Joint Research Projects). The work of Fukui University was partially supported by a Grant-in-Aid from the Ministry of Education, Science and Culture of Japan.

Numerical calculations were performed at the National Institute for Fusion Science Computer Center.

\section{References}

BRAND, G. F., FEKETE, P. W. IDEHARA, T., and MoOre, K. J., 1990, Quasi-optical antennas for plasma scattering. International Joumal of Electronics, 68, 1063-1073.

Fremte, P. W., BrAND, G. F., and IDEHARA, T., 1994, Scattering from discrete Alfven waves in a tokamak using a gyrotron radiation source. Plasma Physics and Controlled Nuclear Fusion, 36, $1407-1417$.

ldehara, T., Shimizu, Y., IChikawa, K., Makino, S., Shubutani, K., Kurahashi, K., Tatsukawa, T., OGaWA, I., OKazaki, Y, and OKamoto, T., 1995, Development of a medium power, submillimeter wave gyrotron using a $17 \mathrm{~T}$ superconducting magnet. physics of plasmas, $2,3246-3248$.

Idehara, T., Tatsukawa, T, OGawa, I., Tanabe, H., Mori, T., Wada, S., Brand, G. F., and BRENNAN, M. H., 1992, Development of a second cyclotron harmonic gyrotron operating at submillimeter wavelengths. Physics of Fluids, A, 267-273.

Ogawa, I., Idehara, T., Pereyaslavets, M., and Kasparek, W., 1999a, Design of a quasioptical system converting the $\mathrm{TE}_{06}$ output mode of a gyrotron into a Gaussian-like beam. International Journal of Infrared and Millimeter Waves, 20, 543-558.

OGaWA, I., SAKai, A., IDeHaRA, T., Kawahata, K., and Kasparek, W., 1997, A quasi optical transmission line for plasma scattering measurements using a submillimeter wave gyrotron. International Journal of Electronics, 83, 635-644.

Ogawa, I., Sakai, A., Idehara, T., and Kasparek, W., 1999b, Application of the complex beam parameter to the design of quasi-optical transmission line for a submillimeter wave gyrotron. International Joumal of Electronics, \$6, 1071-1084.

SpIRA-HAKKARAInEn, S., KReISCher, E. K., and TemkIn, R. J., 1990, Submillimeter-wave harmonic gyrotron experiment. IEEE Transactions on Plasma Science, 18, 334-342.

Suvorov, E. V., Holzhauer, E., Kasparek, W., Lubyako, L. V., Burov, A. B., Dryagin, Y. A., Fiz'chenkov, S. E., Fraiman, A. A., Kukin, L. M., Kostrov, A. V., Ryndyk, D. A., Shtanyuk, A. M., Skalyga, N. K., Smolyaova, O. B., Erckmann, V., Geist, T., KICk, M., LAQuA, H., and Rust, M., 1997, Collective Thomson scattering at WA-AS Plasma Physics and Controlled Fusion, 39, B337-B351.

Terumichi, Y., Kubo, S., Ando, A., Yanagimoto, Y., Ogura, K., TanakA, H., Takahashi, J., Tonai, I., Nakamura, M., Maekawa, T., Tanaka, S., and IDerara, T., 1984, Study of low frequency density fluctuations in the WT-2 tokamak by MM and 
SUBMM wave scattering. Proceedings of 9 th International Conference on Infrared and Millimeter Waves, Takarazuka, 1984, pp. 411-412.

VLASOV, S. N., and ORLovA, I. M., 1974, Quasioptical transformer which transforms the waves in a waveguide having a circular cross section into a highly directional wave beam. Radiofizika, 17, 115-119.

WADA, O., and NAKAJMA, M., 1986, Reflector antennas for electron cyclotron resonance heating of fusion plasma. Space Power, 6, 213-220.

Zaytsev, N. I., Pankratova, T. B., Petelin, M. I., and Flyagin, V. A., 1974, Millimeter- and submillimeter-wave gyrotrons. Radio Engineering and Electronic Physics, 19, $103-107$. 